\title{
ON THE SUMMABILITY OF THE DIFFERENTIATED FOURIER SERIES
}

\author{
BY DANIEL WATERMAN ${ }^{1}$
}

Dedicated to Professor A. Zygmund on the occasion of his 65 th birthday

Communicated by H. Helson, July 21, 1966

A classical theorem of Fatou [2, p. 99] asserts that if $f \in L(0,2 \pi)$ and the symmetric derivative of $f$ at $x_{0}$,

$$
f_{s}^{\prime}\left(x_{0}\right)=\lim _{h \rightarrow 0}\left[f\left(x_{0}+h\right)-f\left(x_{0}-h\right)\right] / 2 h
$$

exists, then the differentiated Fourier series of $f$ is Abel summable to $f_{s}^{\prime}\left(x_{0}\right)$ at $x_{0}$, or equivalently, if $u(r, x)=a_{0} / 2+\sum\left(a_{k} \cos k x+b_{k} \sin k x\right) r^{k}$ is the associated harmonic function, then

$$
\lim _{r \rightarrow 1-0} u_{x}\left(r, x_{0}\right)=f_{s}^{\prime}\left(x_{0}\right) \text {. }
$$

Let us suppose that $\phi$ is a real nonnegative function on an interval to the right of the origin, that $\phi(0)=0$, and that $\phi(t)=O(t)$ as $t \rightarrow 0$. We say that a set is $\phi$-dense at a point $p$ if

$$
m\left(E^{c} \cap I\right) / \phi(m(I)) \rightarrow 0
$$

as $m(I) \rightarrow 0, I$ an interval containing $p$. If $\phi$ is the identity function, this reduces to ordinary metric density. In the case $\phi(t)=t^{\alpha}$, we will say that $E$ is $\alpha$-dense at $p$. Proceeding in a manner entirely analogous to the classical definition of approximate limit and derivative, we say that

$$
\phi-\lim _{t \rightarrow t_{0}} g(t)=a
$$

if for every $\epsilon>0, E_{3}=\{t|| g(t)-a \mid<\epsilon\}$ is $\phi$-dense at $t_{0}$, and we define the $\phi$-approximate symmetric derivative,

$$
\phi-f_{a p s}^{\prime}\left(x_{0}\right)=\phi-\lim _{h \rightarrow 0}\left[f\left(x_{0}+h\right)-f\left(x_{0}-h\right)\right] / 2 h .
$$

We restrict our attention here to the case of most immediate interest, $\alpha$-density, and prove the following

TheOREM. Suppose $f$ is in $L(0,2 \pi)$, of period $2 \pi$, essentially bounded in a neighborhood of $x_{0}$, and, for some $\alpha \geqq 2, y=\alpha-f_{a p s}^{\prime}\left(x_{0}\right)$. Then the

${ }^{1}$ Supported by National Science Foundation Grant No. GP-3987. 
differentiated Fourier series of $f$ is Abel summable to $y$ at $x_{0}$. The value 2 cannot be replaced by a smaller value nor can essentially bounded be replaced by integrable.

Ikegami [1] has shown that $f_{s}^{\prime}$ cannot be replaced by $f_{a p}^{\prime}$ in Fatou's theorem, even if $f$ is bounded. He introduced

$$
\alpha-f_{a p}^{\prime}\left(x_{0}\right)=\alpha-\lim _{h \rightarrow 0 a p}\left[f\left(x_{\theta}+h\right)-f\left(x_{0}\right)\right] / h
$$

and attempted to show that, for bounded $f$, Fatou's theorem holds with this derivative if $\alpha>4$. His argument, however, contains an error, and when it is corrected yields this result only for $\alpha>5$.

Turning to the proof of our result, we may suppose that $x_{0}=0$, $f(0)=0$, and also $\alpha-f_{a p s}^{\prime}(0)=0$ as in the classical case [2, p. 100-101] For the Poisson kernel,

$$
P(r, t)=\frac{1}{2} \cdot \frac{1-r^{2}}{1-2 r \cos t+r^{2}},
$$

we have the estimates

$$
P(r, t)<C \eta /\left(\eta^{2}+t^{2}\right), \quad\left|P_{t}(r, t)\right|<C \eta t /\left(\eta^{4}+t^{4}\right),
$$

where $\eta=1-r$ and, throughout this paper, $C$ will denote a positive constant not necessarily the same at each occurrence. The first estimate here is well known; the other may be obtained in a similar manner.

We may assume $\alpha=2$, for if $\alpha-f_{a p s}^{\prime}(0)$ exists for some $\alpha>2$, it also exists and has the same value for $\alpha=2$.

There is a $\delta_{0}>0$ and an $M>0$ such that $|f(x)| \leqq M$ a.e. in $\left(-\delta_{0}, \delta_{0}\right)$. Now

$$
u_{x}(r, 0)=-\frac{1}{\pi} \int_{0}^{\pi}(f(t)-f(-t)) P_{t}(r, t) d t
$$

and, for any $\delta \in\left(0, \delta_{0}\right)$, we may partition the interval of integration into $(0, \delta),\left(\delta, \delta_{0}\right)$, and $\left(\delta_{0}, \pi\right)$, denoting the absolute values of the above integral over these intervals by $\mathfrak{I}_{1}, \mathfrak{I}_{2}$, and $\mathscr{I}_{3}$ respectively. We show that these values can be made arbitrarily small by choosing $r$ sufficiently close to 1 .

Clearly

$$
g_{2} \leqq 2 M \int_{\delta}^{\pi}\left|P_{t}(r, t)\right| d t<C_{\eta \delta^{-2}}
$$

and 


$$
\begin{aligned}
\mathscr{I}_{3} & \leqq \int_{\delta_{0}}^{\pi}|f(t)-f(-t)|\left|P_{t}(r, t)\right| d t \\
& <C \eta \int_{\delta_{0}}^{\pi} \frac{|f(t)-f(-t)|}{t^{3}} d t<C \eta .
\end{aligned}
$$

Given an $\epsilon>0$, we set

$$
E=\{t||[f(t)-f(-t)] / 2 t \mid \geqq \epsilon\} .
$$

Then

$$
\mathscr{I}_{1} \leqq\left|\int_{E \cap(0, \delta)}^{\ldots \ldots}\right|+\left|\int_{E^{\circ} \cap(0, \delta)}^{\ldots}\right|=\mathscr{g}_{1}^{\prime}+\mathscr{g}_{1}^{\prime \prime}
$$

and we have

$$
g_{1}^{\prime \prime} \leqq \epsilon \int_{0}^{\delta} 2 t\left|P_{t}(r, t)\right| d t<-2 \epsilon \int_{0}^{\pi} t P_{t}(r, t) d t<C \epsilon
$$

by an integration by parts.

The estimation of $\mathcal{I}_{1}^{\prime}$ is somewhat more difficult.

We now choose $\delta$ such that, for $t \in(0, \delta)$,

$$
m(E \cap(0, t))<\varepsilon t^{2}
$$

Let $t_{1}=\delta$ and choose $t_{k}, k=2,3, \cdots$, in $(0, \delta)$, decreasing and converging to zero. We let $I_{k}=\left(t_{k+1}, t_{k}\right)$. Then

$$
\begin{aligned}
\mathfrak{g}_{1}^{\prime} & <M C \eta \int_{E \cap(0, \delta)} t /\left(\eta^{4}+t^{4}\right) d t \\
& <C \eta \sum m\left(E \cap I_{k}\right) t_{k} /\left(\eta^{4}+t_{k+1}^{4}\right)<C \eta \epsilon \sum t_{k}^{3} /\left(\eta^{4}+t_{k+1}^{4}\right) .
\end{aligned}
$$

Now let $t_{k}=\delta / 2^{k-1}$. It is easily verified that

$$
2^{7} \int_{I_{k}} t^{2} /\left(\eta^{4}+t^{4}\right) d t>t_{k}^{8} /\left(\eta^{4}+t_{k+1}^{4}\right)
$$

for every $k$ and, therefore,

$$
\mathscr{g}_{1}^{\prime}<C \eta \epsilon \int_{0}^{\infty} t^{2} /\left(\eta^{4}+t^{4}\right) d t<C \epsilon
$$

Thus

$$
\left|u_{x}(r, 0)\right|<C\left(\epsilon+\eta+\eta \delta^{-2}\right)<C \epsilon
$$


if $\eta$ is sufficiently small, the constant being independent of the choice of $\epsilon$.

Suppose now that $\alpha \in[1,2)$ and choose $\beta \in(\alpha, 2)$. Let $I_{n}=\left(1 / 2^{n}\right.$, $1 / 2^{n}+1 / 2^{\beta n}$ ) and $E=\cup I_{n}$. Then if $1 / 2^{n}<t \leqq 1 / 2^{n-1}$, there exist positive constants $C$ and $C^{\prime}$ such that

$$
C / 2^{\beta n}<m(E \cap(0, t))<C^{\prime} / 2^{\beta n}
$$

for every $n$. Thus $m(E \cap(0, t))=o\left(t^{\alpha}\right)$ as $t \rightarrow 0$. If $f=\chi_{E}$, the characteristic function of $E$, then for sufficiently small $\epsilon>0$,

$$
\{t||[f(t)-f(-t)] / 2 t \mid \geqq \epsilon\}=E
$$

and so

$$
\alpha=f_{a p s}^{\prime}(0)=0 .
$$

For $0<a<b<\pi / 2$, it may be shown that

$$
-\int_{a}^{b} P_{t}(r, t) d t>C \eta r \frac{(a+b)(b-a)}{\eta^{4}+b^{4}} .
$$

Thus, if $\eta=2^{-k}$, we have

$$
\begin{aligned}
u_{x}(r, 0) & =-\frac{1}{\pi} \sum \int_{I_{n}} P_{t}(r, t) d t>-\frac{1}{\pi} \int_{I_{k+1}} P_{t}(r, t) d t \\
& >C 2^{-(\beta+2) k} /\left(2^{-4 k}+\left(2^{-(k+1)}+2^{-\beta(k+1)}\right)^{4}\right) \\
& >C 2^{(2-\beta) k} \rightarrow \infty
\end{aligned}
$$

as $k \rightarrow \infty$, which shows that values of $\alpha<2$ are inadmissible.

Finally suppose $\alpha \geqq 2, \beta>\alpha$, and define $E$ as above. Now let $f=\sum 2^{(\beta-1) n} \chi_{I_{n}}$. Then $f \in L(0,2 \pi)$ and $\alpha-f_{a p s}^{\prime}(0)=0$. However,

$$
\begin{aligned}
u_{x}(r, 0) & >-\int_{I_{k+1}} 2^{(\beta-1)(k+1)} P_{t}(r, t) d t \\
& >C 2^{(\beta-1)(k+1)} \cdot 2^{(2-\beta) k}=C 2^{k} \rightarrow \infty
\end{aligned}
$$

as $k \rightarrow \infty$, which shows that the requirement of essential boundedness cannot be removed.

\section{REFERENCES}

1. T. Ikegami, On Poisson integrals, Proc. Japan Acad. 37 (1961), 14-17. 1959.

2. A. Zygmund, Trigonometric series, vol. I, Cambridge Univ. Press, New York,

Wayne State University 